new field of materials research, intensely pursued and holding fascinating possibilities for future applications and fundamental studies.

Freeman's work exemplifies his leadership in the emerging area of "computational" materials science, centering on the concept that a supercomputer can be viewed as the equivalent of a growth chamber or molecular-beam epitaxy machine, to synthesize and design new materials, and to gain insights into their behavior and properties. His major role in introducing a class of new and fascinating materials complements his numerous other distinguished contributions to the development of materials research.

\section{Duward F. Shriver}

While polymer electrolytes were first studied in Europe, Professor Shriver's laboratory produced the first comprehensive synthetic characterization of themleading to completely new insights into their structure and the mechanisms of ionic transport within them. After successfully developing a continuum elastomeric network interpretation of the mobility mechanism in simple polymer/salt complexes based on polyethylene oxide, Shriver turned his attention to the preparation of new polymer solid electrolytes with tailored properties. A key achievement was his work on the synthesis and characterization of phosphazine-based polymer electrolytes with oligo ether side chains. The combination of the phosphazine backbone (to yield low glass transition temperature and mobility) with oligo ether side chains (to provide complexation to drive the system thermodynamically, towards the elastomeric conductor) was a triumph of imagination and creative solid-state materials.

Shriver's most recent investigation - of polymer-mixed conductors in which both electronic charge and ionic charge are transferred-presents one of the most challenging problems in understanding how charge transport occurs in disordered systems with mixed conductivity.

\title{
MRS Members Choose 1991 Officers, Councillors
}

MRS members cast their ballots this past September to elect three officers and five councillors. Newly elected to serve the Materials Research Society beginning January 1, 1991 are:

\section{First Vice President}

(President-Elect)

G. Slade Cargill III

Senior Manager, Physical Sciences

Department

IBM T.J. Watson Research Center

Yorktown Heights, New York

\section{Second Vice President}

S. Thomas Picraux

Manager, Surface, Interface, and Ion

Beam Research Department

Sandia National Laboratories

Albuquerque, New Mexico

\section{Treasurer}

\section{Charles B. Duke}

Senior Research Fellow

Xerox Research Laboratories

Webster, New York
Councillors

John C. Bravman

Assistant Professor, Department of

Materials Science and Engineering

Stanford University

Stanford, California

\author{
Gregory C. Farrington \\ Dean, School of Engineering and \\ Applied Science \\ University of Pennsylvania \\ Philadelphia, Pennsylvania
}

Julia M. Phillips

Supervisor, Thin Film Research Group

AT\&T Bell Laboratories

Murray Hill, New Jersey

\section{Rustum Roy}

Director, Technology and Society Program

Pennsylvania State University

University Park, Pennsylvania
Carl V. Thompson

Associate Professor, Department of Materials Science and Engineering

Massachusetts Institute of Technology Cambridge, Massachusetts

James B. Roberto, associate director of the Solid State Division at Oak Ridge National Laboratory, who was elected first vice president last year, will serve as president of the Materials Research Society in 1991. Carol M. Jantzen, a ceramist in the Glass Technology Group at the Westinghouse Savannah River Co., will continue to serve her two-year term as treasurer through 1991.

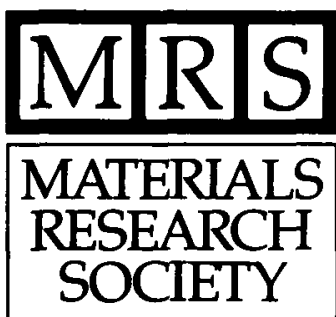




\section{How Thin \\ Do You Need \\ YOUR \\ Silicon \\ Membranes?}

\section{$2-4 \mu$}

ULTRATHIN $^{\mathrm{TM}}$ single crystal wafers are made to your exact thickness requirements.

These elastic membranes are available in:

- 1" thru 4" diameter

- $\langle 100\rangle$ orientation

- $\mathbf{N}$ or P-type doping, or undoped

- As thin as 2-4 $\mu$

For more information about these Ultrathin $^{\mathrm{TM}}$ membranes . . .

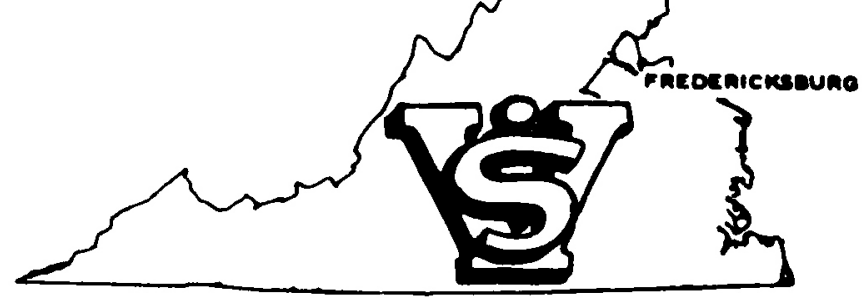
call the leader!

\section{ULTRATHIN $^{\mathrm{TM}}$}

Flat \& Parallel

Membranes

Made to

\section{YOUR}

\section{Specifications}

\section{VIRGINIA SEMICONDUCTOR, INC.}

1501 Powhatan Street, Fredericksburg, VA 22401

Phone (703) 373-2900 • Fax (703) 371-0371

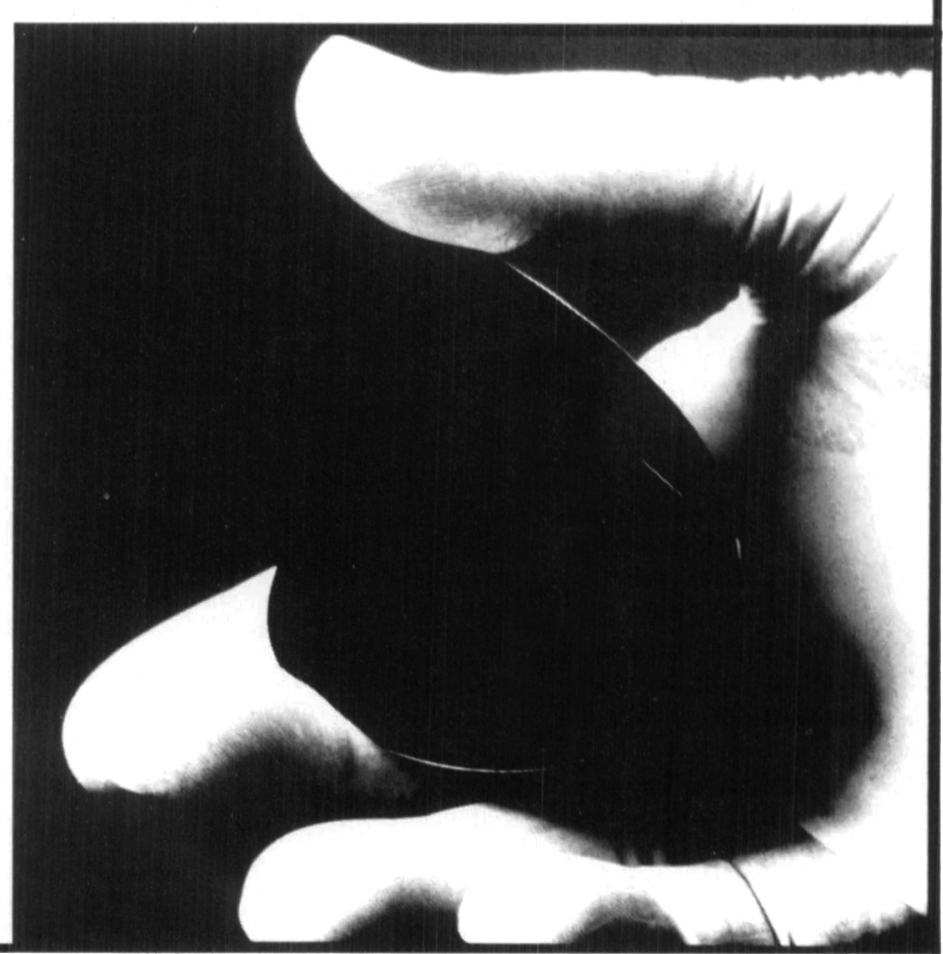


Place your order now for proceedings from the 1990 MRS Fall Meeting in Boston and you'll recolve your books as scon as they are publistred. Special pre-publication prices effective through March 1, 1991. Call (412) 367.3012 to order.

MRS Members, and Fall Moeting \& short course attendees are eligible for MRS member prices.

Surteo Chemistry and Beam-Solid Intornetions

Editors: H. Atwater, F.A. Houle, D. Lowndes

ISBN: 1-55899-093-3 Code: 201B

$\$ 34.00$ MRS Members $\$ 39.00$ US List $\$ 44.00$ foreign

November 26-

December 1 1990

Evolution of Thin Film and Surtace Mierestructure

Editors: C.V. Thompson, J.Y. Tsao, D.J. Srolovitz
ISBN: 1-55899-0941 Code: 202B

$\$ 52.00$ MRS Members \$57.00 US List \$64.00 Foreign

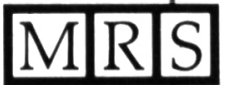

MATERIALS RESEARCH SOCIETY

Boston Marriott Copley Place

Boston,

Massachusetts

Electronic Packoging Motorials Sclence V

Editors: E.D. Lillie, R.J. Jaccodine, P. Ho, K. Jackson ISBN: 1-55899-095-X Code: 2038 ISBN: 1-55899-096-8 Code: 204B

\section{Kinetics of Phase Trantormations} $\$ 20.00$ MRS Members $\$ 35.00$ US List $\$ 42.00$ Foreign

Chemleal Perspectives of Microelectronte Materiels II Editors: L.H. Dubois, L.V. Interrante, M.E. Gross, K.F. Jensen $\$ 37.00$ MRS Members \$42.00 US List \$49.00 Foreign

Editors: M.O. Thompson, M.J. Aziz, G.B. Stephenson, D. Cherns ISBN: 1-55899-097-6 Code: 205B

$\$ 35.00$ MRS Members $\$ 40.00$ US List $\$ 47.00$ Foreign

Clustors and Cluster-Assembled Matorials

Editors: R.S. Averback, D.L. Nelson, J. Bemholc

ISBN: 1-55899008-4 Code: 206B

$\$ 35.00$ MRS Members \$40.00 US List \$47.00 Foreign

Mechanical Properties of Porous and Celluler Motorlats

Editors: L.J. Gibson, D. Green, K. Sieradzdi

ISBN: 1-55899-099-2 Code: 207B

$\$ 25.00$ MRS Members $\$ 30.00$ US List $\$ 37.00$ Foreign

Avrances in Surtaco and Thin Film Difirrection

Editors: P.I. Cohen, D.J. Eaglesham, T.C. Huang

ISBN: 1-55899-100-X Code: 208B

$\$ 30.00$ MRS Members $\$ 35.00$ US List $\$ 40.00$ Foreign

\section{Dobets in Mabriats}

Editors: P.D. Bristowe, J.E. Epperson, J.E. Griffitith, Z. Lilienta-Weber ISBN: 1-55899-101-8 Code: 2098

$\$ 45.00$ MRS Members $\$ 50.00$ US List $\$ 57.00$ Foreign

\section{Solid Stom lonires It}

Editors: G.A. Nazri, R.A. Huggins, D.F. Shriver, M. Balkanskj ISBN: 1-55899-102-6 Code: 2108

$\$ 35.00$ MRS Members $\$ 40.00$ US List $\$ 47.00$ Foreign

Fiber-Roinforeed Comentillous Materlats

Editors: S. Mindess, J.P. Skalny

ISBN: 1-55899-103-4 Code: 211B

$\$ 30.00$ MRS Members $\$ 35.00$ US List $\$ 42.00$ Foreign

Sclentilic Basis tor Nucloar Whato Management XN

Editors: T. Abrajano, Jr. L.H. Johnson

ISBN: 1-55899-1042 Code: 212B

$\$ 45.00$ MRS Members \$50.00 US List \$57.00 Foreign

High Tompercture Ordered Intermetallic Aloys N

Editors: L. Johnson, D.P. Pope, J.O. Stiegler

ISBN: 1-55899-105-0 Code: 213B

$\$ 45.00$ MRS Members $\$ 50.00$ US List $\$ 57.00$ Foreign
Optical and Electrical Properties of Polymers

Editors: J.A. Emerson, J.M. Torkelson

ISBN: 1-55899-106-9 Code: $214 B$

$\$ 28.00$ MRS Members $\$ 33.00$ US List $\$ 40.00$ foreign

Structure, Ralacation and Phydeal folng of

Glassy Polymers

Editors: R.J. Roe, J.M. O'Reilly, J. Torkelson

ISBN: 1-55899-107-7 Code: 2158

$\$ 28.00$ MRS Members $\$ 33.00$ US List $\$ 38.00$ Foreign

Long-Wrowlength Somiconductur Devices,

Matorials and Proceseses

Editors: A. Katz, R.M. Biefeld, R.J. Malik, R.L. Gunshor

ISBN: 1-55899-108-5 Code: 216B

$\$ 32.00$ MRS Members $\$ 38.00$ US List $\$ 45.00$ Foreign

Advanced Tomographic Imaging Mothods for the Analycis of Matorlats

Editors: J.L. Ackerman, W.A. Ellingson

ISBN: 1-55899-109-3 Code: 217B

$\$ 32.00$ MRS Members $\$ 37.00$ US List \$44.00 Forrign

Matorlals Symtheeis Based on Biological Proceses Editors: M. Alper, P.C. Rieke, R. Frankel, P.D. Calvert,

D.A. Tirrell

ISBN: 1-55899-110-7 Code: 218B

$\$ 33.00$ MRS Members $\$ 38.00$ US List $\$ 45.00$ Foreign

\section{EXTENDED ABSTRMCTS}

Electronic, Opticel and Device Propertes of Layered Structures

Editors: J. Hayes, M.S. Hybertsen, E.R. Weber

Code EA-21B

$\$ 15.00$ MRS Mermbers $\$ 20.00$ US List $\$ 22.00$ foreign

Dymanics in Small Confining Syctems

Editors: J.M. Drake, R. Kopelman, J. Klafter

Code EA-22B

\$15.00 MRS Members \$20.00 US List \$22.00 Foreign

Covalent Coranics

Editors: G.S. Fischman, T. Aselage, R.M. Spriggs

Code EA-23B

$\$ 15.00$ MRS Members $\$ 20.00$ US List $\$ 22.00$ Foreign

Synthesis and Proportios of Now Cotalyda: Utilbation of Nowil Materiats Components and Synthotic Trehniques Editors: E.W. Corcoran, Jr., M.J. Ledoux, J.R. Knox Code EA-24B

$\$ 15.00$ MRS Members $\$ 20.00$ US List $\$ 22.00$ Forelgn

Scaling in Disordered Matorials

Editors: J.P. Stokes, M.O. Robbins, T.A. Witten

Code EA-25B

\$15.00 MRS Members \$20.00 US List \$22.00 Foretion

Nanostructures: Fabrication and Physice

Editors: T.P. Smith III, S.D. Berger, D. Kem, H. Craighead

Code EA26B

$\$ 15.00$ MRS Members $\$ 20.00$ US List $\$ 22.00$ Foreign

Call MRS at (412) 367-3012 to place your order today or FAX your order in: (412) 367-4373. Visa, MasterCard and Diners Club accepted, PA residents must pay $6 \%$ sales tax. (Write us at: Materials Research Society, Publications !Dept., 9800 McKnight Road, Pittsburgh, PA 15237, USA) 


\section{0 \\ fall

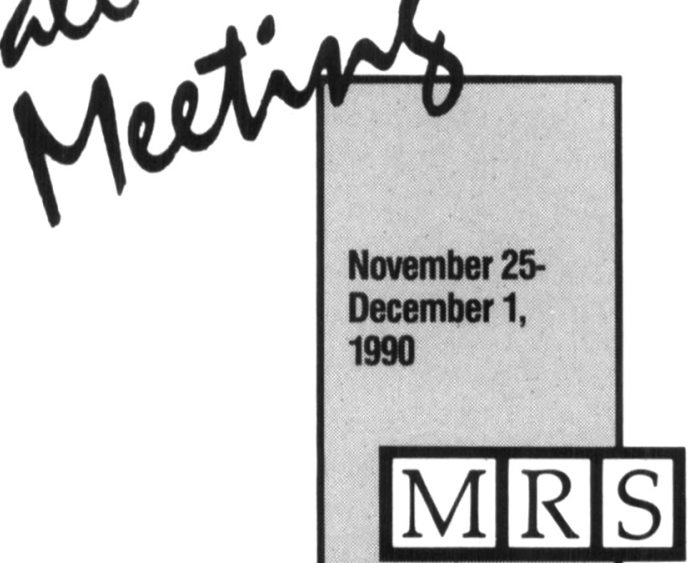 \\ Telephone \\ (412) $367-3003$ \\ $\operatorname{FAX}(412)$ 367-4373

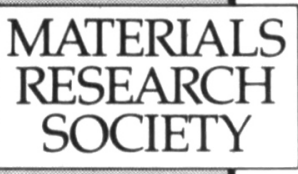 \\ Boston Marriott \& Westin Hotels/ Copley Place Boston, Massachusetts}

PREREGISTRATION

TUITION

\section{ADVANCED MATERIALS}

M-04 Optoelectronic Materials, Processes, and Devices

Instructor: Mool C. Gupta

Friday and Saturday, November 30-December Superconducting Materials

Instructors: Terry P. Orlando and Robert E. Schwall

Sunday and Monday, November 25-26

M-10 An Introduction to High Temperature Ordered Intermetallic Alloys NEW Instructor: Norman S. Stoloff

Monday, November 26

PREPARATION AND FABRICATION OF MATERIALS

P-02 Molecular Beam Epitaxy

Instructor: Gary Wicks

Tuesday and Wednesday, November 27-28

P-03 Vapor Phase Epitaxy

Instructors: Herbert H. Cox and P. Dan Dapkus

Friday and Saturday, November 30-December 1

P-14 Film Formation, Adhesion, Surface Preparation, and Characterization of Thin Film Structures

Instructor: Donald M. Mattox

Friday and Saturday, November 30-December 1

P-20 Growth of Long-Wavelength Detector Materials

NEW Instructors: L. Ralph Dawson, Sorab K. Ghandi, Sanghamitra Sen, and Tse Tung Thursday, Friday and Saturday, November 29-December 1

F-01 Film and Coating Deposition Techniques

Instructor: Donald M. Mattox

Tuesday and Wednesday, November 27-28

F-02 Plasma Etching for Microelectronic Fabrication

Instructor: G. Kenneth Herb

Thursday, November 29

\section{Short Course Program SEVEN NEW COURSE TOPICS}

Selected Short Courses covering the latest developments in materials science and technology will be offered in conjunction with the 1990 Fall Meeting of the Materials Research Society. These up-to-date courses are at the forefront of science and technology and complement Fall Meeting symposium topics. SPECIALTY, REVIEW, AND SURVEY courses are designed to meet needs of professional scientists, engineers, technical staff, and managers who want to know the latest techniques in characterization and fabrication of materials. CLASS SIZES ARE LIMITED: Early telephone preregistrations are encouraged.

F-03 Fundamentals and Applications of Ion Beam Processes Instructor: James K. Hirvonen

Sunday and Monday, November 25-26

F-04 Microelectronic Packaging: Materials, Processing, and Reliability Instructor: Shankara K. Prasad

Thursday, Friday and Saturday, November 29-December 1

F-11 Materials and Processes for Microfeature Fabrication

NEW Instructor: Gary N. Taylor Monday, November 26

F-12 Spin-On Dielectrics for State-of-the-Art VLSI Applications

NEW Instructors: Nadia Lifshitz and Gerald Smolinsky Monday, November 26

TECHNIQUES

T-09 Low Temperature Testing of Superconductors and Semiconductors Instructor: Robert E. Schwall

NEW Tuesday, November 27

CHARACTERIZATION OF MATERIALS

C-01 Modern Materials Analysis Techniques

Instructors: James A. Borders, Kenneth H. Eckelmeyer, and Suzanne H. Weissman Monday, Tuesday and Wednesday November 26-28

C-03 Surface and Thin Film Analysis Instructors: Leonard C. Feldman and James W. Mayer Friday and Saturday, November 30-December 1.

C-09 Fractals: Concepts and Applications in Materials Science and Engineering

Instructors: James E. Martin and Alan J. Hurd Sunday and Monday, November 25-26.

C-14 Fundamentals and Applications of Scanning Tunneling Microscopy Instructor: Robert J. Hamers Monday, November 26

C-17 Scanning, Transmission and Analytical Electron Microscopy Instructors: Alton D. Romig, Jr., and David C. Joy Monday, Tuesday and Wednesday, November 26-28

C-18 TEM Specimen Preparation in the Physical Sciences

NEW Instructor: Ronald M. Anderson Thursday, November 29

C-20 Optical Characterization of III-V Semiconductor Epitaxial Layers Instructor: Gary W. Wicks Thursday, November 29

C-22 Thin Film Epitaxy, Interdiffusion, and Phase Transformation NEW Instructors: Leonard C. Feldman, James W. Mayer, and King-Ning Tu

Thursday and Friday, November 29-30.

\section{SPECIAL DISCOUNTS:}

There are special discounted tuition fees for specific course combinations: M-05 and T-09 total fee is $\$ 775$; F-01 and P-14 - total fee is $\$ 895 ; \mathrm{C}-17$ and C-18 - total fee is $\$ 960$.

Facilities registering three or more persons at the same time in one short course receive a $20 \%$ discount for the third and all additional persons.

\section{MRS ON-SITE SHORT COURSE PROGRAM}

Available at your facility

One of the best ways to keep your staff up to date on the latest developments is through an on-going program of continuing education. Many of the courses described in this flyer, as well as others not being presented at the 1990 Fall Meeting, are now available on a contract basis for presentation at your facility or technical meeting.

For further details about courses available at your facility, nearby site, or your technica

Vivienne Harwood Mattox, MRS Short Course Manager; 440 Live Oak Loop,

Albuquerque, NM 87122; (505) 294-9532, FAX (505) 298-7942

$\$ 345$
REGISTRATION INFORMATION: Call (412) 367-3003 and ask for the Short Course

Office to request information about student scholarships and special meeting registration discounts. 


\section{EQUIPMENT EXHIBIT \\ 1990 MRS Fall Meeting \\ Boston Marriott \\ Copley Place Hotel \\ Tuesday-Thursday, November 27-29}

As part of the 1990 MRS Fall Meeting, a major equipment exhibit will display analytical and processing equipment closely paralleling the nature of the technical symposia. The technical program has been arranged to allow meeting participants ample opportunity to visit the exhibit, which will be located on the third floor of the Boston Marriott Copley Place Hotel.

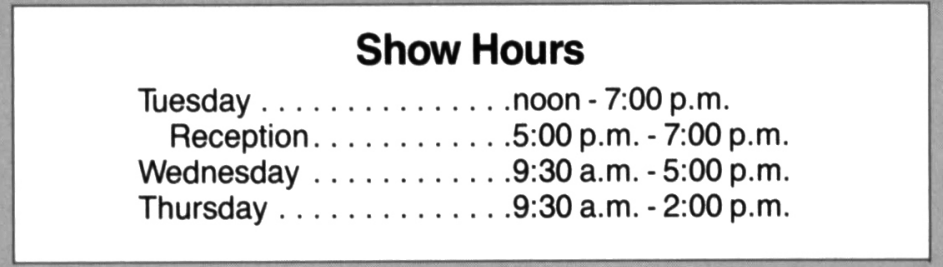

\section{EXHIBITORS \\ (as of September 19, 1990)}

Academic Press, Inc.

AG Associates

Aixtron Inc.

American Chemical Society

American Institute of Physics

AMER-TEM

Anatech Ltd.

APD Cryogenics, Inc.

Applied Science and Technology/ASTEX

\section{Balzers}

Blake Industries, Inc.

Butterworth-Heineman

Cahn Instruments

Cambridge Molecular Design

Cambridge University Press

Cameca Instruments, Inc.

Ceramaseal

Chapman and Hall

Commonwealth Scientific Corporation

Cryomech, Inc.

Denton Vacuum, Inc.

Digital Instruments

EG\&G Parc

ElectroScan Corporation

Elsevier Science Publishers

Emcore Corporation

Energy Beam Sciences, Inc.

EPI

Charles Evans \& Associates

FEI Company

E.A. Fischione Instruments

Ernest F. Fullam, Inc.

Gatan Inc.

Geller MicroAnalytical Laboratory

Goodfellow Corporation

Granville-Phillips Co.

R.G. Hansen \& Associates

Hiden RGA Division
Hitachi Scientific Instruments

Huntington Labs

lbis Technology

Image Micro Systems, Inc.

Implant Sciences Corporation

INEL, Inc.

Innovative Technology, Inc.

Instron Corporation

Instruments SA/JY Optical Division

Instruments SA/Riber Division

International Scientific Instruments

Ion Tech, Inc.

Janis Research Company

JCPDS-ICDD

JEOL U.S.A.

Keithley Instruments

Kimball Physics Inc.

Kratos Analytical

Lake Shore Cryotronics

Lambda Physik

Kurt J. Lesker Co.

Leybold Vacuum Products

Link Analytical

Luxtron Corporation

Maxwell Electronics

McAllister Technical Services

MDC Vacuum Products Corporation

Microscience, Inc.

MKS Instruments, Inc.

MMR Technologies

MR Semicon, Inc.

National Electrostatics Corporation

Nor-Cal Products, Inc.

North Eastern Analytical Corporation

Omicron Associates

Oxford Instruments NA, Inc.

Oxford Plasma Technology

Oxford University Press
Peabody Scientific

Pergamon Press

Perkin-Elmer Corporation

Philips Electronic Instruments Company

Plasma-Therm

Plenum Publishing Corporation

Princeton Gamma-Tech, Inc.

Princeton Instruments

Process Products Corporation

Pure Tech Inc.

Quantum Design, Inc.

Rigaku/USA

RMC Cryosystems

Rudolph Research

Scientific Instruments

Scintag Buehler

Siemens X-Ray

SOPRA

South Bay Technology, Inc.

Spectra Instruments

SPEX Industries, Inc.

Spire Corporation

Springer-Verlag N.Y.

Strem Chemicals Inc.

Structure Probe, Inc./SPI Supplies

Superconductive Components, Inc.

SYCON Instruments

Tencor Instruments

Thermionics Laboratory Inc.

Ulvac/Sinku Riku

VG Instruments

Vacuum Barrier Corporation

Varian/Vacuum Products Division

VAT, Inc.

VCR Group, Inc.

Virginia Semiconductor, Inc.

Voltaix, Inc.

Wavemat

Wyko Corporation

Carl Zeiss, Inc. 
Advanced Control Systems Corporation

Advanced Energy Industries, Inc.

Advanced Micro Devices, Inc.

Aerospace Corporation

AET addax, Inc.

AG Associates

Air Products - Diamonex

Aixtron $\mathrm{GmbH}$

Alcan International Limited

Alcatel NV

Alcoa

Allied-Signal, Inc.

American Cyanamid Company

Amoco Corporation

Amoco Chemical Corporation

Amoco Oil Co.

Amoco Technology Company

Anatech Ltd.

APD Cryogenics Inc

APL Engineered Materials, Inc.

Applied Electron Corporation

Applied Materials, Inc.

Applied Science and Technology, Inc. (ASTeX)

Argonne National Laboratory/IPNS

Asahi Glass Company, Ltd.

AT\&T Bell Laboratories

Barbeau-Hutchings Advertising

Bell Communications Research, Inc.

Billiton Precursors B.V.

Bio-Rad Laboratories

Blake Industries, Inc.

BP America Research \&

Development

Brimrose Corporation of America

British Telecom

Brookhaven National Laboratory

Bruker Instruments Inc.

Butterworths Scientific Ltd.

Cabot Corporation

California Institute of Technology

Cameca Instruments, Inc.

Center for Materials Fabrication

Chronar Corporation

Cober Electronic, Inc.

Coherent Laser Products Group

Commonwealth Scientific Corporation

Conversion Technology Corporation

Corning Glass Works

CrystaComm, Inc.

Crystallume

CVC Products, Inc

David Sarnoff Research Center

Denton Vacuum Inc.

Deposition Technology

Diamond Materials, Inc.

Digital Instruments, Inc.

Doty Scientific, inc

Dow Chemical Company

Dow Corning Corporation

E.I. duPont de Nemours \& Company

Eastman Kodax Company

Eaton Corporation

EG\&G Idaho, Inc.

EG\&G Princeton Applied Research

Electric Power Research Institute

(EPRI)
Elettrorava S.p.A.

Elsevier Science Publishers B.V.

Emcore Corporation

Engelhard Corporation

EPI Division Chorus Corporation

Epitronics Corporation

Charles Evans \& Associates

Exxon Basic Chemicals Technology

Exxon Research \& Engineering Co.

Evans East, Inc.

FEI Company

E.A. Fischone Instruments Manulacturing

Flexus Incorporated

Ford Motor Company

Forest Products Laboratory

Friendship United Corporation

Fuji Electric Co., Itd.

Fuji Xerox Co., Ltd.

Fujitsu Ltd.

Galileo Corporation of America

Gas Research Institute

Gatan, Inc.

Gelest Inc.

General Electric Corp./Advanced Inorganic Materials Lab.

General Electric Corp./Aircraft Engines Division

General Electric Corporation

General Motors Research Laboratories

Gerling Laboratories

Getty Conservation Institute

Glasstech Solar, Inc. (GSI)

Goodfellow Corporation

Granville-Phillips Company

GTE Laboratories, Inc.

Heraeus Amersil

Hewlett-Packard, NMD

High Voltage Engineering Europa B.V.

Hitachi Research Lab.

Hitachi Scientific Instruments

Hoechst Celanese Research Division

Hoya Optics, Inc.

HTR Sciences

Hughes Research Laboratories

Huntington Laboratories

IBM Corporation

IBM Japan, Ltd.

ICI Polyurethanes

Imperial Chemical Industries

Innovative Technology, Inc

INSPEC/IEE

Instron Corporation

Instruments S.A., Inc./Riber Division

International Centre for Diffraction Data (JCPDS)

International Scientific Instruments, inc.

Ion Tech, Inc.

lowa Fly Ash

James River Corporation

Janis Research Company, Inc.

JEOL U.S.A., Inc.

Johnson \& Johnson Orthopaedics

Kanegafuchi Chemical Industry Co., Ltd.

Kennametal, Inc.
Kluwer Academic Publishers

Kobe Development Corporation

Kogaku Giken Company, Ltd.

Kopin Corporation

Kratos Analytical, Inc.

Kyocera Corporation

Lake Shore Cryotronics, Inc.

Lam Research Corporation

Lambda Physik, Inc.

Lawrence Livermore National

Laboratory

Kurt J. Lesker Company

Leybold Inficon Inc.

Leybold Vacuum Products, Inc.

Los Alamos National Laboratory

Manics

Martin Marietta Energy Systems, Inc.

Martin Marietta Laboratories

Materials Research Corporation

Matheson Gas Products

Matsushita Electrical Industrial Co.

MDC Vacuum Products Corporation

MEMC Electronic Materials Inc.

Microelectronics \& Computer

Technology Corporation (MCC)

Micromap

Microscience, Inc.

Mitsui Petrochemical Industries, Ltd.

MKS Instruments, Inc.

Mobay Corporation

Mobil Research \& Development Corporation

Molycorp, Inc. (a Unocal Company)

Monsanto Company

MR Semicon, Inc.

Nano Instruments, Inc.

NASA Lewis Research Center

National Electrostatics Corporation

National Semiconductor

NEC Research Institute Inc.

Nimic, Inc.

Nippon Denso Co., Ltd.

Nippon Mining Company, L.td.

Nippon Telegraph \& Telephone

Corporation

Nissei Sangyo America

NIST

North Eastern Analytical

Corporation

Northern Telecom Electronics Ltd.

Norton Company

Oak Ridge National Laboratory

OIS, Inc. (Ovonic Imaging Systems Inc.)

Ortech International

Oxford Instruments North America

Pacific Northwest Laboratory

Peak Systems, Inc.

Pergamon Press, Inc.

Perkin-Elmer

Pfizer, Inc.

Philips Electronic Instruments

Plasma Sciences, Inc.

PPG Industries Glass R\&D Center

PQ Corporation

The Proctor \& Gamble Company

Prometrix Corporation
Quantum Design

Raychem Corporation

Raytheon Company

Rhone-Poulenc Inc

Rigaku USA Inc.

Rockwell International Science Center

Sandia National Laboratories

Sanyo Electric Co., Ltd.

Schlumberger-Doll Research

Schott Fiber Optics, Inc.

Scienta Instruments AB

Sharp Corporation

Siemens Analytical X-Ray Instruments, Inc.

Siemens Solar Industries

Solar Energy Research Institute (SERI)

Solarex Corporation

Solecon Laboratories, Inc.

Solid State Measurements, Inc.

South Bay Technology, Inc.

Spex Industries, Inc.

Spire Corporation

Springer-Verlag New York, Inc.

SSC, Inc.

Strem Chemicals, Inc

Sumitomo Electric USA, Inc.

Sumitomo Metal Mining Co., Ltd.

Superconductive Components, Inc.

Superconductivity Publications, Inc.

Surface Science Instruments, Inc.

Tamarack Scientific Co., Inc.

Texas Instruments, Inc.

3M Company

Toei Industry Co., Ltd.

Tonen Corporation

Toshiba Corporation

Tosoh Corporation

Tracor Northern

Ultra High Vacuum Instruments Inc.

Ultratherm Inc.

Union Carbide Chemical \& Plastics Co.

United Technologies Research Center

Universal Energy Systems

UOP Corporation

USG Research Center

Vacuum/Atmospheres Company

Vacuum Barrier Corporation

Varian Assocs., Inc./Continental Electronic Div.

Varian Assocs., Inc./Extrion Div.

Varian Assocs., Inc./Thin Film

Technology Div.

VCH Publishers, Inc.

VG Instruments, Inc.

VG Microscopes Ltd.

Voltaix, Inc.

Wacker Siltronic Corporation

Wavemat Inc.

Westinghouse Electric Corporation

W.R. Grace \& Company

W.R. Grace \& Co./Research and

Davison Chemical Div.

Xerox Corporation

Carl Zeiss, Inc. 